Ladislav Šamaj

\title{
Finite-Size Effects in Non-Neutral Two-Dimensional Coulomb Fluids
}

Received: / Accepted:

\begin{abstract}
Thermodynamic potential of a neutral two-dimensional (2D) Coulomb fluid, confined to a large domain with a smooth boundary, exhibits at any (inverse) temperature $\beta$ a logarithmic finite-size correction term whose universal prefactor depends only on the Euler number of the domain and the conformal anomaly number $c=-1$. A minimal free boson conformal field theory, which is equivalent to the $2 \mathrm{D}$ symmetric two-component plasma of elementary $\pm e$ charges at coupling constant $\Gamma=\beta e^{2}$, was studied in the past. It was shown that creating a non-neutrality by spreading out a charge $Q e$ at infinity modifies the anomaly number to $c(Q, \Gamma)=-1+3 \Gamma Q^{2}$. Here, we study the effect of non-neutrality on the finite-size expansion of the free energy for another Coulomb fluid, namely the 2D one-component plasma (jellium) composed of identical pointlike $e$-charges in a homogeneous background surface charge density. For the disk geometry of the confining domain we find that the non-neutrality induces the same change of the anomaly number in the finite-size expansion. We derive this result first at the free-fermion coupling $\Gamma \equiv \beta e^{2}=2$ and then, by using a mapping of the $2 \mathrm{D}$ one-component plasma onto an anticommuting field theory formulated on a chain, for an arbitrary coupling constant.
\end{abstract}

Keywords Coulomb fluid · Finite-size correction · Central charge · Conformal field theory

\section{Introduction}

According to the principle of conformal invariance [1,2,3, two-dimensional (2D) systems of constituents with short-range interactions, confined to a

Institute of Physics, Slovak Academy of Sciences, Dúbravská cesta 9, SK-84511 Bratislava, Slovakia

E-mail: Ladislav.Samaj@savba.sk 
large domain with a smooth boundary, exhibit at the critical point universal finite-size properties. In particular, for a disk domain of radius $R, D=\{\mathbf{r} \in$ $\left.\mathbb{R}^{2},|\mathbf{r}| \leq R\right\}$ with the Euler number $\chi=1$, the (dimensionless) free energy has the large- $R$ expansion

$$
\beta F=(\beta f) \pi R^{2}+(\beta \sigma) 2 \pi R-\frac{c}{6} \ln \left(\frac{R}{L}\right)+O(1),
$$

where $\beta \equiv 1 /\left(k_{\mathrm{B}} T\right)$ is the inverse temperature and $L$ is a length scale. The bulk specific free energy $\beta f$ and the surface tension $\beta \sigma$ are non-universal, i.e., dependent on microscopic details of the given model and on the temperature. The logarithmic term is universal, depending only on the conformal anomaly number (central charge) $c$ of the critical theory. Like for instance, the massless Gaussian theory with a field $\phi(\mathbf{r})$, defined by the Hamiltonian

$$
H_{\mathrm{G}}=\frac{1}{4 \pi} \int_{D} \mathrm{~d}^{2} r(\nabla \phi)^{2},
$$

has $c=1$ [4.

In this paper, we study $2 \mathrm{D}$ classical (i.e., non-quantum) systems of charged pointlike particles interacting pairwisely by the long-range Coulomb potential $\phi(\mathbf{r})=-\ln (|\mathbf{r}| / L)$, where the dielectric constants of the medium in which particles move and of the walls are equal to 1 (vacuum in Gauss units), for simplicity. The logarithmic potential is the solution of the 2D Poisson equation

$$
\Delta \phi(\mathbf{r})=-2 \pi \delta(\mathbf{r})
$$

and in three dimensions corresponds to the effective interaction of infinitely long parallel charged lines, perpendicular to the plane. Two types of Coulomb systems are of special interest. The one-component plasma (OCP), or jellium, is a system of identical mobile (say elementary) charges $e$ immersed in a homogeneous neutralizing background charge density. The symmetric two-component plasma (TCP), or the Coulomb gas, is a system of oppositely charged $\pm e$ mobile particles with no background. While the OCP is usually studied in the canonical ensemble, the TCP is treated within the grand-canonical ensemble, with the enforced neutrality condition for each microscopic configuration. In both cases, the thermodynamics and the particle correlation functions are determined by the only dimensionless parameter, the coupling constant $\Gamma=\beta e^{2}$. The $2 \mathrm{D}$ OCP is exactly solvable at $\Gamma=2$ by mapping onto free fermions 17 . The 2D TCP undergoes the collapse of positive-negative pairs of pointlike charges at coupling $\Gamma=2$, which corresponds to the exactly solvable free-fermion point of the equivalent Thirring model 5, 14. For reviews about exactly solvable 2D Coulomb systems with various geometries of confining domains, see Refs. 12,19.

At arbitrary temperature of the conducting regime, the long-range tail of the Coulomb potential induces screening. Due to the screening effect, the bulk particle correlations exhibit short-range (exponential or even Gaussian) decay which indicates a non-criticality. On the other hand, the same screening phenomenon causes that the induced electrical-field correlations are longranged [21,29. As a result, the free energy (or the grand potential) of any 
Coulomb system exhibits a universal finite-size correction of type (1.1). Since the logarithmic Coulomb potential is the inverse of the Laplacian operator $\Delta$, in the functional sense it is associated with the Gaussian Hamiltonian (1.2) rewritten as $-\int_{D} \mathrm{~d}^{2} r \phi \Delta \phi /(4 \pi)$. Although the conformal anomaly number of Coulomb systems is expected to be related to the Gaussian one, there is a change of sign, namely

$$
c=-1
$$

The explicit checks of the universal finite-size behavior with $c=-1$ were done, for both OCP and TCP, at the exactly solvable coupling $\Gamma=2$. The studied cases involve Coulomb gases with periodic boundary conditions [11, confined to a domain by plain hard walls 20 , by ideal-conductor boundaries 22 and by ideal-dielectric walls 26,43 . For Coulomb systems living on a surface of the sphere, a direct derivation of the universal finite-size correction was performed for any coupling by combining stereographic projection of the sphere onto an infinite plane with linear response theory (TCP, Ref. [23]) or with density functional approach (OCP, Ref. [25]). The prefactor to the universal correction term was related to the second moment of the short-range part of the planar direct correlation function. Using a renormalized Mayer expansion [7,13 and observing a cancellation property of specific families of renormalized diagrams, this second moment was evaluated for both TCP [24] and OCP 28. Another explicit derivation of the universal logarithmic term at an arbitrary coupling constant was made for the TCP in a disk geometry 35]. All obtained results confirm the conformal prediction (1.1) for a critical system as we had $c=-1$.

All that has been said holds for neutral Coulomb systems. Recently, Ferrero and Téllez 10 studied the $2 \mathrm{D}$ TCP of $\pm e$ charges at the exactly solvable collapse $\Gamma=2$ point, confined to a disk of radius $R$. They fixed a "guest" hard-core impurity of charge $Q e$ at the disk origin and found that the large$R$ expansion for the grand potential of type (1.1) applies if the conformal anomaly number is modified to

$$
c(Q, \Gamma=2)=-1+6 Q^{2}
$$

presence of the impurity has no effect on bulk and boundary terms. It has been noted in the same reference that the same modification of the anomaly number occurs when the minimal free boson conformal field theory [8, 15], equivalent formally to the 2D TCP, is deformed by spreading out a charge $Q e$ at infinity, creating in this way a non-neutral system. The equivalence of the two systems, one with a charge fixed at the disk origin and one with the same charge spread out at infinity, can be explained by noting that a charge fixed at the disk origin is screened at microscopic distances by counterions from the TCP and thus the universal large- $R$ correction looks like the one for a non-neutral system with the net charge $Q e$. Notice that the definition of $c$ in Refs. [8, 10] has opposite sign with respect to the standard notation used in this paper. At an arbitrary $\Gamma$, conformal field theory [8, 15] yields

$$
c(Q, \Gamma)=-1+3 \Gamma Q^{2}
$$


With this conformal number, the logarithmic correction in (1.1) is no longer universal, its prefactor depends on both the coupling constant and the excess charge.

In this paper, we concentrate on the non-neutral 2D OCP confined to the disk. We perform a microscopic derivation of the finite-size expansion of the free energy and find that it is of type (1.1) with the same anomaly number (1.6) as found for the 2D TCP in the context of conformal field theories. We would like to note that in contrast to the previous studies [27,30,31] of charge fluctuations in finite Coulomb systems in a much larger overall neutral system or with particle reservoir put at infinity, here we consider non-neutral systems within the constrained canonical ensemble with the fixed net charge and look for the dependence of finite-size corrections of the free energy on this excess charge.

The paper is organized as follows. In Sect. 2, we derive the total energy of mobile charged particles and the fixed background in presence of nonneutrality. Section 3 summarizas the general mapping of the 2D OCP onto the theory of anticommuting variables on a one-dimensional chain. The exactly solvable free-fermion case is the subject of Sect. 4. The free-fermion result is generalized to an arbitrary coupling in Sect. 5. In Sect. 6, we give a short recapitulation and some concluding remarks.

\section{Derivation of Boltzmann factor}

Let us consider the 2D OCP inside the disk $D$ of radius $R$. There are $N$ pointlike particles of charge $e$ immersed in a uniform background charge density $\rho_{b}=-e n_{b}$, where the background density $n_{b}$ is given by

$$
\pi R^{2} n_{b}=N-Q, \quad \text { finite } Q<N
$$

The net charge of the system is thus $Q e$ and the neutral case corresponds to $Q=0$. The particle density $n$ is given by

$$
\pi R^{2} n=N
$$

As

$$
\frac{n_{b}}{n}=1-\frac{Q}{N}
$$

the particle and background densities coincide in the thermodynamic limit $N \rightarrow \infty$.

The potential induced by the background charge density reads as

$$
V(\mathbf{r})=-\int_{D} \mathrm{~d}^{2} r^{\prime} \rho_{b} \ln \left|\frac{\mathbf{r}-\mathbf{r}^{\prime}}{L}\right|
$$

This potential satisfies the Poisson equation with circular symmetry

$$
\Delta V(\mathbf{r}) \equiv \frac{1}{r} \frac{\partial}{\partial r}\left[r \frac{\partial}{\partial r} V(r)\right]=2 \pi e n_{b}
$$


The potential at the disk boundary is given by the total background charge placed at the origin, i.e., $V(R)=e n_{b}\left(\pi R^{2}\right) \ln (R / L)$. The solution of Eq. (2.5), supplemented by this boundary condition, reads

$$
V(r)=e n_{b} \pi\left[\frac{r^{2}}{2}+R^{2} \ln \left(\frac{R}{L}\right)-\frac{R^{2}}{2}\right] .
$$

To derive the total Boltzmann factor, we proceed in close analogy with neutral systems, see e.g. Refs. 6, 12, 41. The total Coulomb interaction energy of the particle-background system consists of three parts:

- the particle-particle interaction

$$
\begin{aligned}
U_{1} & =-e^{2} \sum_{1 \leq j<k \leq N} \ln \frac{\left|\mathbf{r}_{j}-\mathbf{r}_{k}\right|}{L} \\
& =\frac{e^{2}}{2} N(N-1) \ln L-e^{2} \sum_{1 \leq j<k \leq N} \ln \left|\mathbf{r}_{j}-\mathbf{r}_{k}\right|,
\end{aligned}
$$

- the particle-background interaction

$$
\begin{aligned}
U_{2} & =e \sum_{j=1}^{N} V\left(\mathbf{r}_{j}\right) \\
& =\frac{e^{2} n_{b} \pi}{2} \sum_{j=1}^{N} r_{j}^{2}+e^{2} N(N-Q)\left[\ln \left(\frac{R}{L}\right)-\frac{1}{2}\right],
\end{aligned}
$$

- the background-background interaction

$$
\begin{aligned}
U_{3} & =-\frac{1}{2} \int_{D} \mathrm{~d}^{2} r \int_{D} \mathrm{~d}^{2} r^{\prime} \rho_{b}^{2} \ln \left|\frac{\mathbf{r}-\mathbf{r}^{\prime}}{L}\right| \\
& =-\frac{e^{2}}{2}(N-Q)^{2}\left[\ln \left(\frac{R}{L}\right)-\frac{1}{4}\right] .
\end{aligned}
$$

With the definition of the coupling constant $\Gamma=\beta e^{2} \equiv 2 \gamma$, the corresponding Boltzmann factor reads as

$$
\begin{aligned}
\mathrm{e}^{-\beta\left(U_{1}+U_{2}+U_{3}\right)}= & \exp \left[\gamma Q^{2} \ln \left(\frac{R}{L}\right)+\gamma N \ln L-\gamma N^{2} \ln R+\frac{3}{4} \gamma N^{2}\right. \\
& \left.-\frac{1}{2} \gamma N Q-\frac{1}{4} \gamma Q^{2}\right] \prod_{j=1}^{N} \mathrm{e}^{-\pi \gamma n_{b} r_{j}^{2}} \prod_{1 \leq j<k \leq N}\left|\mathbf{r}_{j}-\mathbf{r}_{k}\right|^{2 \gamma} \cdot(2.10)
\end{aligned}
$$

From the expression for the canonical partition function

$$
Z_{\gamma}(N, Q)=\frac{1}{N !} \int_{D} \prod_{j=1}^{N} \frac{\mathrm{d}^{2} r_{j}}{\lambda^{2}} \mathrm{e}^{-\beta\left(U_{1}+U_{2}+U_{3}\right)},
$$


the free energy $F_{\gamma}(N, Q)$ is given by $-\beta F_{\gamma}(N, Q)=\ln Z_{\gamma}(N, Q)$, i.e.,

$$
\begin{aligned}
-\beta F_{\gamma}(N, Q)= & \gamma Q^{2} \ln \left(\frac{R}{L}\right)+\gamma N \ln L-\gamma N^{2} \ln R+\frac{3}{4} \gamma N^{2} \\
& -\frac{1}{2} \gamma N Q-\frac{1}{4} \gamma Q^{2}-2 N \ln \lambda+\ln I_{\gamma}(N, Q) .
\end{aligned}
$$

Here,

$$
I_{\gamma}(N, Q)=\frac{1}{N !} \int_{D} \prod_{j=1}^{N}\left[\mathrm{~d}^{2} r_{j} w\left(\mathbf{r}_{j}\right)\right] \prod_{1 \leq j<k \leq N}\left|\mathbf{r}_{j}-\mathbf{r}_{k}\right|^{2 \gamma}
$$

is the configuration integral with the circularly symmetric one-body Boltzmann factor

$$
w(\mathbf{r})=\exp \left(-\gamma \pi n_{b} r^{2}\right) .
$$

\section{Mapping onto 1D fermions}

There exist two basic approaches how to treat the configuration integral (2.13). The first one is based on the expansion of the power of van der monde determinants in the basis of Jack polynomials 32,42,44. The second one is based on the mapping onto a 1D lattice fermion system; this formalism has been introduced in Ref. [33] and developed further in Refs. [34, 36, 37, 38, 39, 40. The relation between the two methods was established in Ref. [16]. Here, we apply the mapping onto $1 \mathrm{D}$ fermions.

For $\gamma$ a positive integer, the configuration integral (2.13) can be expressed in terms of two sets of anticommuting variables $\left\{\xi_{j}^{(\alpha)}, \psi_{j}^{(\alpha)}\right\}$ each with $\gamma$ components $(\alpha=1, \ldots, \gamma)$, defined on a discrete chain of $N$ sites $j=0,1, \ldots, N-1$, as follows

$$
I_{\gamma}=\int \mathcal{D} \psi \mathcal{D} \xi \mathrm{e}^{\mathcal{S}(\xi, \psi)}, \quad \mathcal{S}(\xi, \psi)=\sum_{j=0}^{\gamma(N-1)} w_{j} \Xi_{j} \Psi_{j}
$$

Here, $\mathcal{D} \psi \mathcal{D} \xi \equiv \prod_{j=0}^{N-1} \mathrm{~d} \psi_{j}^{(\gamma)} \cdots \mathrm{d} \psi_{j}^{(1)} \mathrm{d} \xi_{j}^{(\gamma)} \cdots \mathrm{d} \xi_{j}^{(1)}$ and the action $\mathcal{S}(\xi, \psi)$ involves pair interactions of composite operators

$$
\Xi_{j}=\sum_{\substack{j_{1}, \ldots, j_{\gamma}=0 \\\left(j_{1}+\cdots+j_{\gamma}=j\right)}}^{N-1} \xi_{j_{1}}^{(1)} \cdots \xi_{j_{\gamma}}^{(\gamma)}, \quad \Psi_{j}=\sum_{\substack{j_{1}, \ldots, j_{\gamma}=0 \\\left(j_{1}+\cdots+j_{\gamma}=j\right)}}^{N-1} \psi_{j_{1}}^{(1)} \cdots \psi_{j_{\gamma}}^{(\gamma)},
$$

i.e. the products of $\gamma$ anticommuting variables from a given set with the fixed sum of site indices. The elements of the diagonal interaction matrix are given by

$$
w_{j}=\int_{D} \mathrm{~d}^{2} r r^{2 j} w(r), \quad j=0,1, \ldots, \gamma(N-1) .
$$


For the present one-body Boltzmann factor (2.14), the interaction strengths can be rewritten as follows

$$
w_{j}=\frac{1}{\gamma n_{b}} \frac{1}{\left(\gamma \pi n_{b}\right)^{j}} \tilde{w}_{j}, \quad \tilde{w}_{j}=\int_{0}^{\gamma(N-Q)} \mathrm{d} t t^{j} \mathrm{e}^{-t} .
$$

The one-body density of particles at point $\mathbf{r} \in D$ is defined by

$$
n(\mathbf{r})=\langle\hat{n}(\mathbf{r})\rangle, \quad \hat{n}(\mathbf{r})=\sum_{j=1}^{N} \delta\left(\mathbf{r}-\mathbf{r}_{j}\right),
$$

where $\hat{n}(\mathbf{r})$ is the microscopic density of particles at point $\mathbf{r}$ and $\langle\cdots\rangle$ denotes the statistical average over canonical ensemble. The one-body density can be obtained from the configuration integral (2.13) in the standard way as the functional derivative:

$$
n(\mathbf{r})=w(\mathbf{r}) \frac{1}{I_{\gamma}} \frac{\delta I_{\gamma}}{\delta w(\mathbf{r})} .
$$

Within the formalism of anticommuting variables, the circularly symmetric one-body density is expressible explicitly as

$$
n(r)=w(r) \sum_{j=0}^{\gamma(N-1)}\left\langle\Xi_{j} \Psi_{j}\right\rangle r^{2 j}
$$

where

$$
\langle\cdots\rangle \equiv \frac{1}{I_{\gamma}} \int \mathcal{D} \psi \mathcal{D} \xi \mathrm{e}^{S} \ldots
$$

denotes averaging over anticommuting variables.

\section{The free-fermion point $\Gamma=2$}

For $\Gamma=2(\gamma=1)$, the composite operators (3.2) are the ordinary anticommuting variables. The diagonalized action in (3.1) then implies

$$
I_{1}(N, Q)=\prod_{j=0}^{N-1} w_{j}
$$

Since the correlators

$$
\left\langle\Xi_{j} \Psi_{j}\right\rangle=\frac{1}{w_{j}}, \quad j=0,1, \ldots, N-1,
$$

the particle density (3.7) takes the form

$$
n(N, Q ; r)=\mathrm{e}^{-\pi n_{b} r^{2}} \sum_{j=0}^{N-1} \frac{r^{2 j}}{w_{j}} .
$$


According to (3.4), the interaction strengths are expressible as

$$
w_{j}=\frac{1}{n_{b}} \frac{1}{\left(\pi n_{b}\right)^{j}} \tilde{w}_{j}, \quad \tilde{w}_{j}=\int_{0}^{N-Q} \mathrm{~d} t t^{j} \mathrm{e}^{-t} .
$$

Since

$$
\ln I_{1}(N, Q)=-N \ln n_{b}-\frac{1}{2} N(N-1) \ln \left(\pi n_{b}\right)+\sum_{j=0}^{N-1} \ln \tilde{w}_{j},
$$

the expression (2.12) for the free energy can be rewritten as

$$
\begin{aligned}
-\beta F_{1}(N, Q)= & Q^{2} \ln \left(\frac{R}{L}\right)+\frac{1}{2} N \ln \left(\pi n_{b} L^{2}\right)-\frac{1}{2} N^{2} \ln (N-Q) \\
& +\frac{3}{4} N^{2}-\frac{1}{2} N Q-\frac{1}{4} Q^{2}-N \ln \left(n_{b} \lambda^{2}\right)+\sum_{j=0}^{N-1} \ln \tilde{w}_{j} .
\end{aligned}
$$

Now we aim at estimating the crucial sum $\sum_{j=0}^{N-1} \ln \tilde{w}_{j}$. Since it holds

$$
\tilde{w}_{j}=\int_{0}^{N} \mathrm{~d} t t^{j} \mathrm{e}^{-t}-\mathrm{e}^{-N} \int_{0}^{Q} \mathrm{~d} s(N-s)^{j} \mathrm{e}^{s}
$$

we get the important relation

$$
\frac{\partial \tilde{w}_{j}}{\partial Q}=-\mathrm{e}^{-(N-Q)}(N-Q)^{j}
$$

Consequently,

$$
\begin{aligned}
\frac{\partial}{\partial Q} \sum_{j=0}^{N-1} \ln \tilde{w}_{j} & =-\mathrm{e}^{-(N-Q)} \sum_{j=0}^{N-1} \frac{1}{\tilde{w}_{j}}(N-Q)^{j} \\
& =-\frac{1}{n_{b}} \mathrm{e}^{-(N-Q)} \sum_{j=0}^{N-1} \frac{1}{w_{j}}\left(\frac{N-Q}{\pi n_{b}}\right)^{j} \\
& =-\frac{1}{n_{b}} \mathrm{e}^{-\pi n_{b} R^{2}} \sum_{j=0}^{N-1} \frac{R^{2 j}}{\tilde{w}_{j}}=-\frac{n(N, Q ; R)}{n_{b}},
\end{aligned}
$$

where $n(N, Q ; R)$ is the particle number density at $r=R$, i.e., at the wall contact. We can write

$$
\sum_{j=0}^{N-1} \ln \tilde{w}_{j}=\sum_{j=0}^{N-1} \ln j !+\sum_{j=0}^{N-1} \ln \left(\frac{1}{j !} \int_{0}^{N} \mathrm{~d} t t^{j} \mathrm{e}^{-t}\right)-\int_{0}^{Q} \mathrm{~d} Q^{\prime} \frac{n\left(N, Q^{\prime} ; R\right)}{n_{b}} .
$$

This formula completes the determination of the free energy (4.6).

Let us now consider the large- $N$ limit when the background density is related to the particle density via $n_{b}=n+O(1 / N)$. We start with the sum 
representation (4.10). With the aid of formulas presented in Appendix A of Ref. [6], we find that

$$
\sum_{j=0}^{N-1} \ln j !=\frac{1}{2} N^{2} \ln N-\frac{3}{4} N^{2}+N \frac{\ln (2 \pi)}{2}-\frac{1}{12} \ln N+O(1) .
$$

Using the asymptotic formula 9

$$
\frac{1}{j !} \int_{0}^{N} \mathrm{~d} t t^{j} \mathrm{e}^{-t}=\frac{1}{2}\left[1+\Phi\left(\frac{N-n}{\sqrt{2 N}}\right)\right]+O\left(\frac{1}{\sqrt{N}}\right)
$$

with

$$
\Phi(u)=\frac{2}{\sqrt{\pi}} \int_{0}^{u} \mathrm{~d} v \mathrm{e}^{-v^{2}}
$$

being the error function, we obtain $[20$.

$$
\sum_{j=0}^{N-1} \ln \left(\frac{1}{j !} \int_{0}^{N} \mathrm{~d} t t^{j} \mathrm{e}^{-t}\right)=\sqrt{2 N} \int_{0}^{\infty} \mathrm{d} v \ln \left[\frac{1+\Phi(v)}{2}\right]+O(1) .
$$

Finally, the integral

$$
\int_{0}^{Q} \mathrm{~d} Q^{\prime} \frac{n\left(N, Q^{\prime} ; R\right)}{n_{b}}
$$

depends on the statistical average $n\left(N, Q^{\prime} ; R\right)$ which is finite for any $N$, and therefore in the limit $N \rightarrow \infty$ contributes to $O(1)$. It is natural to suppose that in the limit $N \rightarrow \infty$ the statistical averages do not depend on the finite charge imbalance $Q^{\prime} e$ within an infinite domain and so the particle density at the disk border $n\left(N, Q^{\prime} ; R\right)$ tends to its half-space "wall contact" value $n_{\text {wall }}=n \ln 2$ [18, independent of $Q^{\prime}$. Thus we have

$$
\lim _{N \rightarrow \infty} \int_{0}^{Q} \mathrm{~d} Q^{\prime} \frac{n\left(N, Q^{\prime} ; R\right)}{n_{b}}=Q \ln 2
$$

which is the term of order $O(1)$. To summarize,

$$
\begin{aligned}
\sum_{j=0}^{N-1} \ln \tilde{w}_{j}= & \frac{1}{2} N^{2} \ln N-\frac{3}{4} N^{2}+N \frac{\ln (2 \pi)}{2}-\frac{1}{12} \ln N \\
& +\sqrt{2 N} \int_{0}^{\infty} \mathrm{d} v \ln \left[\frac{1+\Phi(v)}{2}\right]+O(1) .
\end{aligned}
$$

Inserting this large- $N$ formula into (4.6) and taking into account that

$$
-\frac{1}{2} N^{2} \ln (N-Q)-\frac{1}{2} N Q=-\frac{1}{2} N^{2} \ln N+O(1),
$$


we arrive at the finite-size expansion of type (1.1) with

$$
\begin{aligned}
\beta f & =n\left[\ln \left(n \lambda^{2}\right)-\frac{1}{2} \ln \left(\pi n L^{2}\right)-\frac{1}{2} \ln (2 \pi)\right], \\
\beta \sigma & =-\sqrt{\frac{n}{2 \pi}} \int_{0}^{\infty} \mathrm{d} v \ln \left[\frac{1+\Phi(v)}{2}\right], \\
c(Q, \Gamma=2) & =-1+6 Q^{2} .
\end{aligned}
$$

We see that for the coupling $\Gamma=2$ the central charge depends on $Q$ in the same way as for the $2 \mathrm{D}$ TCP at the same coupling constant $\Gamma=2$, see Eq. (1.5).

\section{Arbitrary $\Gamma=2 \gamma$ with integer $\gamma$}

For an arbitrary integer $\gamma$, there exist certain analogies with the exactly solvable $\gamma=1$ case.

In the configuration integral (3.1), we perform the unitary transformation of anticommuting variables, say $\xi$ 's,

$$
\xi_{j} \rightarrow \mu^{1 / \gamma} \lambda^{j} \tilde{\xi}_{j}, \quad \Xi_{j} \rightarrow \mu \lambda^{j} \tilde{\Xi}_{j},
$$

which keeps the composite form of $\Xi$-operators. Here, $\mu$ and $\lambda$ are free asyet unspecified parameters. Taking into account the corresponding Jacobian, this transformation modifies $I_{\gamma}(N, Q)$ to the form

$$
I_{\gamma}(N, Q)=\mu^{-N} \lambda^{-\gamma N(N-1) / 2} \tilde{I}_{\gamma}(N, Q),
$$

where

$$
\tilde{I}_{\gamma}(N, Q)=\int \mathcal{D} \psi \mathcal{D} \tilde{\xi} \exp \left(\sum_{j=0}^{\gamma(N-1)} \mu \lambda^{j} w_{j} \tilde{\Xi}_{j} \Psi_{j}\right)
$$

Choosing

$$
\mu=\gamma n_{b}, \quad \lambda=\gamma \pi n_{b}
$$

we eliminate the $Q$-dependent prefactor in (3.4) and in this way pass from $w_{j}$ to $\tilde{w}_{j}$. We obtain that

$$
\ln I_{\gamma}(N, Q)=-N \ln \left(\gamma n_{b}\right)-\frac{\gamma}{2} N(N-1) \ln \left(\gamma \pi n_{b}\right)+\ln \tilde{I}_{\gamma}(N, Q),
$$

where

$$
\tilde{I}_{\gamma}(N, Q)=\int \mathcal{D} \psi \mathcal{D} \tilde{\xi} \exp \left(\sum_{j=0}^{\gamma(N-1)} \tilde{w}_{j} \tilde{\Xi}_{j} \Psi_{j}\right) .
$$

To obtain a convenient representation of $\ln \tilde{I}_{\gamma}(N, Q)$, we differentiate this quantity with respect to $Q$ :

$$
\frac{\partial}{\partial Q} \ln \tilde{I}_{\gamma}(N, Q)=\sum_{j=0}^{\gamma(N-1)}\left\langle\tilde{\Xi}_{j} \Psi_{j}\right\rangle \frac{\partial \tilde{w}_{j}}{\partial Q} .
$$


Since

$$
\tilde{w}_{j}=\int_{0}^{\gamma N} \mathrm{~d} t t^{j} \mathrm{e}^{-t}-\gamma \mathrm{e}^{-\gamma N} \int_{0}^{Q} \mathrm{~d} s[\gamma(N-s)]^{j} \mathrm{e}^{\gamma s},
$$

we get

$$
\frac{\partial \tilde{w}_{j}}{\partial Q}=-\gamma \mathrm{e}^{-\gamma(N-Q)}[\gamma(N-Q)]^{j}
$$

As concerns the correlators $\left\langle\tilde{\Xi}_{j} \Psi_{j}\right\rangle$, we return back to the original anticommuting $\xi$ 's variables by applying the inverse to the transformation (5.1). The Jacobians of the numerator and the denominator cancel with one another and we obtain

$$
\left\langle\tilde{\Xi}_{j} \Psi_{j}\right\rangle=\frac{1}{\mu \lambda^{j}}\left\langle\Xi_{j} \Psi_{j}\right\rangle=\frac{1}{\gamma n_{b}} \frac{1}{\left(\gamma \pi n_{b}\right)^{j}}\left\langle\Xi_{j} \Psi_{j}\right\rangle
$$

Consequently, the relation (5.7) takes the form

$$
\begin{aligned}
\frac{\partial}{\partial Q} \ln \tilde{I}_{\gamma}(N, Q) & =-\frac{1}{n_{b}} \mathrm{e}^{-\gamma(N-Q)} \sum_{j=0}^{\gamma(N-1)}\left\langle\Xi_{j} \Psi_{j}\right\rangle\left(\frac{N-Q}{\pi n_{b}}\right)^{j} \\
& =-\frac{1}{n_{b}} \mathrm{e}^{-\gamma \pi n_{b} R^{2}} \sum_{j=0}^{\gamma(N-1)}\left\langle\Xi_{j} \Psi_{j}\right\rangle R^{2 j}=-\frac{n(N, Q ; R)}{n_{b}}
\end{aligned}
$$

As the result

$$
\ln \tilde{I}_{\gamma}(N, Q)=\ln \tilde{I}_{\gamma}(N, Q=0)-\int_{0}^{Q} \mathrm{~d} Q^{\prime} \frac{n\left(N, Q^{\prime} ; R\right)}{n_{b}} .
$$

Putting together Eqs. (2.12), (5.5) and (5.12), the free energy is given by

$$
\begin{aligned}
\beta F_{\gamma}(N, Q)= & -\gamma Q^{2} \ln \left(\frac{R}{L}\right)-\frac{1}{2} \gamma N \ln \left(\gamma \pi n_{b} L^{2}\right)+\frac{1}{2} \gamma N^{2} \ln [\gamma(N-Q)] \\
& -\frac{3}{4} \gamma N^{2}+\frac{1}{2} \gamma N Q+\frac{1}{4} \gamma Q^{2}+N \ln \left(\gamma n_{b} \lambda^{2}\right) \\
& -\ln \tilde{I}_{\gamma}(N, Q=0)+\int_{0}^{Q} \mathrm{~d} Q^{\prime} \frac{n\left(N, Q^{\prime} ; R\right)}{n_{b}}
\end{aligned}
$$

As before, the mean particle density at the disk border $n\left(N, Q^{\prime} ; R\right)$ is finite for any $N$ and in the large- $N$ limit we have

$$
\lim _{N \rightarrow \infty} \int_{0}^{Q} \mathrm{~d} Q^{\prime} \frac{n\left(N, Q^{\prime} ; R\right)}{n_{b}}=Q \frac{n_{\text {wall }}}{n} .
$$

In contrast to the $\gamma=1$ case, the explicit value of $n_{\text {wall }} / n$ is not known, but the important information is that this term is of order $O(1)$. Consequently,

$$
\beta F_{\gamma}(N, Q)=\beta F_{\gamma}(N, Q=0)-\frac{\Gamma}{2} Q^{2} \ln \left(\frac{R}{L}\right)+O(1) .
$$


Considering for the neutral $\beta F_{\gamma}(N, Q=0)$ the anticipated critical finite-size expansion (1.1) with $c=-1$, the conformal anomaly number is equal to the one predicted by conformal field theory in Eq. (1.6).

Although the proof was made for the 2D OCP with $\gamma$ a positive integer, it is reasonable to extend its validity to all real coupling values $\Gamma>0$, within the fluid regime.

\section{Conclusion}

This work was motivated by the recent paper of Ferrero and Téllez [10] about the finite-size expansion of the grand potential for the $2 \mathrm{D}$ TCP at the coupling $\Gamma=2$, in the presence of the impurity. The modification of the universal logarithmic term to the non-universal one, dependent on the charge $Q e$ of impurity, was there in agreement with the prediction (1.5) of non-neutral conformal field theories.

In this paper, we tested the prediction (1.5) of conformal field theory on the non-neutral 2D OCP. At the free-fermion coupling $\Gamma=2$ (Sect. 4), we reproduce the result (1.5). Using the mapping of the $2 \mathrm{D}$ OCP onto the anticommuting field theory formulated on the chain, we were able to extend the finite-size analysis to an arbitrary coupling constant $\Gamma=2 *$ integer. This is one of rare occasions when the exact results are obtained for a series of pair-integer $\Gamma$ values. The non-universal prediction of conformal field theory (1.6) is confirmed as well.

It would be interesting to generalize our results to the 2D TCP or even to Coulomb fluids with an arbitrary charge composition. For such systems, we miss techniques analogous to that for 2D OCP, but a phenomenological approach might reveal the general form of the finite-size logarithmic term.

This work might be a further motivation for specialists to use Coulomb fluids as practical realizations of conformal field theories to test their predictions.

Acknowledgements The support received from Grant VEGA No. 2/0015/15 is acknowledged.

\section{References}

1. Affleck, I.: Universal term in the free energy at a critical point and the conformal anomaly. Phys. Rev. Lett. 56, 746-748 (1986)

2. Blöte, H.W.J., Cardy, J.L., Nightingale, M.P.: Conformal invariance, the central charge, and universal finite-size amplitudes at criticality. Phys. Rev. Lett. 56, 742-745 (1986)

3. Cardy, J.L., Peschel, I.: Finite-size dependence of the free energy in twodimensional critical systems. Nucl. Phys. B 300, 377-392 (1988)

4. Cardy, J.L.: Conformal invariance and statistical mechanics. In: Brézin, E, Zinn-Justin, J. (eds.), Fields, Strings and Critical Phenomena, Les Houches 1988, Session XLIX, North-Holland, Amsterdam (1990)

5. Cornu, F., Jancovici, B.: On the two-dimensional Coulomb gas. J. Stat. Phys. 49, 33-56 (1987) 
6. Di Francesco, P., Gaudin, M., Itzykson, C., Lesage, F.: Laughlin's wave functions, Coulomb gases and expansions of the discriminant. Int. J. Mod. Phys. A 9, 4257-4351 (1994)

7. Deutsch, C., Lavaud, M.: Equilibrium properties of a two-dimensional Coulomb gas. Phys. Rev. A 9, 2598-2616 (1974)

8. Dotsenko, V.S.: Serie de cours sur la théorie conform. Université de Paris VIVII (2004)

9. Erdélyi, A.: Higher Transcendental Functions. McGraw-Hill, New York (1953)

10. Ferrero, A., Téllez, G.: Screening of an electrically charged particle in a twodimensional two-component plasma at $\Gamma=2$. J. Stat. Mech., P11021 (2014)

11. Forrester, P.J.: Finite-size corrections to the free energy of Coulomb systems with a periodic boundary condition. J. Stat. Phys. 63, 491-504 (1991)

12. Forrester, P.J.: Exact results for two-dimensional Coulomb systems. Phys. Rep. 301, 235-270 (1998)

13. Friedman, H.L.: Ionic solution theory. Interscience, New York (1962)

14. Gaudin, M.: Critical isotherm of a lattice plasma. J. Phys. France 46 1027-1042 (1985)

15. Ginsparg, P.: Applied conformal field theory. In: Brézin, E, Zinn-Justin, J. (eds.), Fields, Strings and Critical Phenomena, Les Houches 1988, Session XLIX, North-Holland (1990)

16. Grimaldo, J.A.M., Telléz, G.: Relations among two methods for computing the partition function of the two-dimensional one-component plasma. J. Stat. Phys. 160, 4-28 (2015)

17. Jancovici, B.: Exact results for the two-dimensional one-component plasma. Phys. Rev. Lett. 46, 386-388 (1981)

18. Jancovici, B.: Classical Coulomb systems near a plane wall. I. J. Stat. Phys. 28, 43-65 (1982)

19. Jancovici, B.: Inhomogeneous two-dimensional plasmas. In: Henderson. D. (ed.) Inhomogeneous Fluids, pp. 201-237, Dekker, New York (1992)

20. Jancovici B., Manificat, G., Pisani, C.: Coulomb systems seen as critical systems: Finite-size effects in two dimensions. J. Stat. Phys. 76, 307-329 (1994)

21. Jancovici, B.: Classical Coulomb systems: Screening and correlations revisited. J. Stat. Phys. 80, 445-459 (1995)

22. Jancovici, B., Telléz, G.: Coulomb systems seen as critical systems: Ideal conductor boundaries. J. Stat. Phys. 82, 609-632 (1996)

23. Jancovici, B.: A sum rule for the two-dimensional two-component plasma. J. Stat. Phys. 100, 201-207 (2000)

24. Jancovici, B., Kalinay, P., Samaj, L.: Another derivation of a sum rule for the two-dimensional two-component plasma. Physica A 279, 260-267 (2000)

25. Jancovici, B., Trizac, E.: Universal free energy correction for the twodimensional one-component plasma. Physica A 284, 241-245 (2000)

26. Jancovici, B., Šamaj, L.: Coulomb systems with ideal dielectric boundaries: Free fermion point and universality. J. Stat. Phys. 104, 753-775 (2001)

27. Jancovici: Charge fluctuations in finite Coulomb systems. J. Stat. Phys. 110 879-902 (2003)

28. Kalinay, P., Markoš, P., Šamaj, L., Travěnec, I.: The sixth-moment sum rule for the pair correlations of the two-dimensional one-component plasma: Exact result. J. Stat. Phys. 98, 639-666 (2000)

29. Lebowitz, J.L., Martin, Ph.A.: On potential and field fluctuations in classical charged systems. J. Stat. Phys. 34, 287-311 (1984).

30. Levesque, D., Weis, J.-J., Lebowitz, J.L.: Charge fluctuations in the twodimensional one-component plasma. J. Stat. Phys. 100, 209-222 (2000).

31. Martin, Ph.A., Yalcin, T.: The charge fluctuations in classical Coulomb systems. J. Stat. Phys. 22, 435-463 (1980)

32. Salazar, R., Telléz, G.: Exact energy computation of the one component plasma on a sphere for even values of the coupling parameter, J. Stat. Phys. 164, 969$999(2016)$

33. Šamaj, L., Percus, J.K.: A functional relation among the pair correlations of the two-dimensional one-component plasma. J. Stat. Phys. 80, 811-824 (1995) 
34. Šamaj, L.: Microscopic calculation of the dielectric susceptibility tensor for Coulomb fluids. J. Stat. Phys. 100, 949-967 (2000)

35. Šamaj, L., Jancovici, B.: Density correlations in the two-dimensional Coulomb gas. J. Stat. Phys. 106, 323-355 (2002)

36. Šamaj, L.: Is the two-dimensional one-component plasma exactly solvable? J. Stat. Phys. 117, 131-158 (2004)

37. Šamaj, L., Trizac, E.: Counter-ions at charged walls: Two-dimensional systems. Eur. Phys. J. E 34, 20 (2011)

38. Šamaj, L., Trizac, E.: Counter-ions between or at asymmetrically charged walls: 2D free-fermion point. J. Stat. Phys. 156, 932-947 (2014)

39. Šamaj, L.: Counter-ions near a charged wall: Exact results for disc and planar geometries. J. Stat. Phys. 161, 227-249 (2015)

40. Šamaj, L.: Amplitude function of asymptotic correlations along charged wall in Coulomb fluids. J. Stat. Phys. 164, 304-320 (2016)

41. Sari, R.R., Merlini, D., Calinon, R.: On the ground state of the one-component classical plasma. J. Phys. A: Gen. Phys. 9, 1539-1551 (1976)

42. Telléz, G., Forrester, P.J.: Exact finite-size study of the $2 \mathrm{D}$ OCP at $\Gamma=4$ and $\Gamma=6$. J. Stat. Phys. 97, 489-521 (1999)

43. Telléz, G.: Two-dimensional Coulomb systems in a disk with ideal dielectric boundaries. J. Stat. Phys. 104, 945-970 (2001)

44. Telléz, G., Forrester, P.J.: Expanded Vandermonde powers and sum rules for the two-dimensional one-component plasma. J. Stat. Phys. 148, 824-855 (2012) 